\title{
Effects of SGLT2 inhibitors on eGFR in type 2 diabetic patients-the role of antidiabetic and antihypertensive medications
}

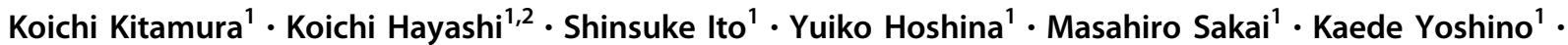 \\ Keita Endo ${ }^{1} \cdot$ Shigeki Fujitani ${ }^{3} \cdot$ Toshihiko Suzuki $^{1}$
}

Received: 18 August 2020 / Revised: 29 September 2020 / Accepted: 20 October 2020 / Published online: 14 December 2020

(c) The Author(s) 2020. This article is published with open access

\begin{abstract}
Recent randomized trials demonstrating the beneficial effects of sodium-glucose cotransporter 2 inhibitors (SGLT2is) in type 2 diabetes suggest that early reductions in eGFR upon initiation of SGLT2i therapy are associated with improved renal outcomes. Multiple concomitant medications, including antidiabetic and antihypertensive agents, are commonly used, however, which may modify the renal hemodynamic action of SGLT2is. Here we found that background treatment with metformin diminished the SGLT2i-induced reductions in eGFR after 3 months of SGLT2i therapy in patients with type 2 diabetes and hypertension $\left(-2.29 \pm 0.90 \mathrm{vs}-5.85 \pm 1.27 \mathrm{~mL} / \mathrm{min} / 1.73 \mathrm{~m}^{2}\right.$ for metformin users $(n=126)$ and nonusers $(n=$ 97), respectively). Other antidiabetic agents (DPP4 inhibitors, sulfonylureas and insulin) had no effect on the eGFR response to SGLT2is. Antihypertensive drugs, including calcium channel blockers (CCBs) and $\beta$ blockers, did not affect the SGLT2iinduced changes in eGFR, whereas renin-angiotensin system inhibitors (RASis) tended to enhance this response $(p=0.059)$. Next, we evaluated the interaction between metformin and RASis in the eGFR responses to SGLT2is. Under no background treatment with RASis, metformin abrogated the eGFR response to SGLT2is, but this response was preserved when RASis had been given along with metformin (decreases of $0.75 \pm 1.28$ vs. $4.60 \pm 1.15 \mathrm{~mL} / \mathrm{min} / 1.73 \mathrm{~m}^{2}$ in eGFR, $p=0.028$ ). No interaction between metformin and insulin or between metformin and DPP4 inhibitors was observed. In conclusion, metformin blunts the SGLT2i-induced decrease in eGFR, but coadministration of RASis ameliorates this response. Furthermore, the inability of CCBs to modify the SGLT2i-induced reduction in eGFR suggests that the SGLT2i-induced renal microvascular action is mediated predominantly by postglomerular vasodilation rather than preglomerular vasoconstriction.
\end{abstract}

Keywords $\mathrm{Ca}^{2+}$ channel blockers $\cdot \mathrm{eGFR} \cdot \operatorname{metformin} \cdot$ renin-angiotensin system inhibitors $\cdot$ renal microvessels $\cdot$ SGLT2 inhibitors

\section{Introduction}

Diabetes mellitus (DM) not only causes detrimental effects on cardiovascular integrity but also aggravates renal function, which should lead to end-stage kidney disease requiring renal replacement therapy. Recent advances in pharmaceutical

Supplementary information The online version of this article (https:// doi.org/10.1038/s41440-020-00590-1) contains supplementary material, which is available to authorized users.

Toshihiko Suzuki

toshihikos@jadecom.jp

1 Tokyo Bay Urayasu Ichikawa Medical Center, Chiba, Japan

2 Department of Internal Medicine, Tokyo Dental College, Chiyoda City, Japan

3 Department of Emergency and Critical Care Medicine, St Marianna University School of Medicine, Kawasaki, Japan sciences facilitate the development of new classes of antidiabetic drugs, including dipeptidyl peptidase 4 (DPP4) inhibitors, glucagon-like peptide-1 (GLP-1) receptor agonists and sodium-glucose cotransporter 2 (SGLT2) inhibitors. Whereas a variety of conventional antidiabetic drugs have been shown to retard the progression of nephropathy through glycemic control [1], there are a limited number of observations demonstrating the direct salutary action of antidiabetic drugs on the progression of nephropathy [2]. Recently, convincing evidence has accumulated that SGLT2 inhibitors offer direct renal protective action, including alleviation of albuminuria and prevention of worsening nephropathy [3-6]. Although it is generally accepted that SGLT2 inhibitors reduce blood pressure (BP) and body weight (BW) along with blood glucose, the alterations in these factors cannot fully explain the renal benefits observed in patients with DM nephropathy, and the mechanisms whereby SGLT2 inhibitors improve renal outcomes are not well understood. Alternatively, it has been 
established that SGLT2 inhibitors cause an acute decrease in estimated glomerular filtration rate (eGFR), which remains stable thereafter and is reversible upon cessation of the therapy $[3,4,6]$. These findings could lend support to the conjecture that the beneficial action of SGLT2 inhibitors is attributed in part to the mitigation of glomerular hypertension, the mechanism of which is allegedly mediated by enhanced tubuloglomerular feedback (TGF) resulting from increased delivery of sodium chloride to the macula densa and causing afferent arteriolar constriction [7].

DM triggers a chain of diseases, including hypertension and dyslipidemia, and requires multiple drug therapies. Hypertension, in particular, constitutes an important therapeutic target because it causes renal damage and accelerates the progression of DM nephropathy. It has been established that renin-angiotensin system (RAS) inhibitors are the treatment of choice because of their unique pharmacological characteristics, i.e., the ability to reduce not only systemic BP but also glomerular pressure. Furthermore, calcium channel blockers (CCBs) are administered as an add-on therapy for strict BP control. Notably, in the EMPA-REG OUTCOME trial, approximately $80 \%$ of the subjects had been given RAS inhibitors, and 27.8-45.8\% received $\mathrm{CCBs}$ as background medications to SGLT2 inhibitors [3]. These findings prompted us to speculate that the renal hemodynamic effects of SGLT2 inhibitors are modified by background medications such as RAS inhibitors and CCBs, both of which possess vasodilator action on renal microvessels. Finally, the treatment of type 2 DM usually requires multiple antidiabetic drugs, among which metformin is recognized as a first-line drug. Intriguingly, recent post hoc analyses of the EMPA-REG OUTCOME [8] and the CANVAS [9] trials have demonstrated that there are greater reductions in risk for worsening nephropathy and heart failure in patients not using metformin than in those using metformin at baseline. Nevertheless, the acute effects of SGLT2 inhibitors on renal function under metformin therapy have not been scrutinized hitherto.

We therefore conducted a retrospective survey evaluating the effect of SGLT2 inhibitors on eGFR over the 12-month study period and examined whether this effect was modified by other antidiabetic and/or antihypertensive drugs in patients with type 2 DM. The present study demonstrates close interplay among SGLT2 inhibitors, metformin and RAS inhibitors, all of which are commonly used together for glycemic and BP control. SGLT2 inhibitor-induced decreases in eGFR, allegedly associated with alleviation of glomerular hypertension, were diminished by metformin, but this response can be restored by further addition of RAS inhibitors. These observations thus merit comprehensive consideration with regard to the renal hemodynamic action of SGLT2 inhibitors and their background medications when SGLT2 inhibitors are used in patients with DM and hypertension.

\section{Methods}

This study is a retrospective analysis evaluating the role of antihypertensive and antidiabetic agents on the renal hemodynamic effects of SGLT2 inhibitors in type 2 DM. The study was approved by the Ethics Committee of Tokyo Bay Urayasu-Ichikawa Medical Center with waiver of the requirement for obtaining informed consent (approval No. 443) and was registered at UMIN (ID; UMIN000037043). The study was conducted in accordance with the Declaration of Helsinki. Information from medical records was anonymized and deidentified prior to final analysis.

\section{Study population}

We enrolled 255 patients with type $2 \mathrm{DM}$ who visited the outpatient clinic of Tokyo Bay Urayasu Ichikawa Medical Center and had been treated with SGLT2 inhibitors between April 2014 and September 2018. Eligible subjects were aged 18 years or older and were either treatment-naïve (i.e., no glucose-lowering drugs) or on antidiabetic therapy before administration of SGLT2 inhibitors with HbA1c between $7.0 \%$ and $11.0 \%$. Patients on steroid therapy or immunosuppressive agents were excluded from the study. Ultimately, 223 subjects were included in this study.

\section{Study design}

The effects of SGLT2 $\mathrm{i}$ on eGFR as well as systolic and diastolic BP were evaluated over 12 months. Because multiple antidiabetic and antihypertensive agents that could affect renal hemodynamics are commonly used as background medications, the effects of these drugs on SGLT2 inhibitor-induced changes in eGFR were also assessed. Biochemical parameters, including HbA1c, serum creatinine, LDL-cholesterol (LDL-C) and serum $\mathrm{Mg}$, as well as anthropometric parameters (BP and $\mathrm{BW}$ ), were assessed over 12 months following the administration of SGLT2 inhibitors. eGFR was calculated using the formula adapted to the Japanese population [10].

$$
\begin{aligned}
\mathrm{eGFR} & =194 \times \text { age }^{-0.287} \\
& \times \text { serum creatinine }{ }^{-1.094}(\times 0.739, \text { if female })
\end{aligned}
$$

\section{Statistical analysis}

The results are expressed as the mean \pm SE or median [lower quartile, upper quartile]. Data were compared by ttest or Mann-Whitney U test, as appropriate. Serial changes in eGFR over 12 months were assessed with a piecewise linear regression model in two time periods: 0 to 3 months and 3 to 12 months. Stepwise multiple regression analyses 
were applied to evaluate which parameters (age, sex, antidiabetic/antihypertensive drugs used, changes in $\mathrm{HbAlc}$, systolic $\mathrm{BP}$ and $\mathrm{BW}$ ) had a greater impact on the early changes ( 0 to 3 months) in eGFR induced by SGLT2 inhibitors. Statistical analyses were performed using Statistical Package for Social Sciences (SPSS) version 25 (IBM, www.ibm.com). P values less than 0.05 were considered statistically significant.

\section{Results}

\section{Effects of SGLT2i on renal hemodynamics}

The baseline characteristics of SGLT2 inhibitor-treated subjects showed that the mean age was $58.1 \pm 0.8 \mathrm{y} / \mathrm{o}$, and HbAlc levels were $7.89 \pm 0.08 \%$ (Table 1). The patients' eGFR was $73.2 \pm 1.6 \mathrm{~mL} / \mathrm{min} / 1.73 \mathrm{~m}^{2}$ (Table 2), and $73.5 \%$ of the subjects exhibited relatively preserved renal function (eGFR $\geq 60 \mathrm{~mL} / \mathrm{min} / 1.73 \mathrm{~m}^{2}$ ).

The following SGLT2 inhibitors were given: dapagliflozin (32.7\%), ipragliflozin (28.3\%), canagliflozin (14.8\%), empagliflozin (11.2\%), luseogliflozin (8.1\%) and tofogliflozin $(4.9 \%)$. Other glucose-lowering drugs used concomitantly with SGLT2 inhibitors are shown in Table 1. Metformin and sulfonylurea were avoided in patients with moderately impaired renal function (Supplementary Table). For BP-lowering agents, RAS inhibitors were given to $51.1 \%$ of the subjects (angiotensin receptor blockers (ARBs); 30.9\%, angiotensin-converting enzyme inhibitors (ACEis); 20.2\%) prior to the administration of SGLT2

Table 1 Baseline characteristics of patients

\begin{tabular}{lllr}
\hline & & Drug therapies & $N(\%)$ \\
\hline$N$ (male/female) & $223(149 / 74)$ & Antidiabetics \\
Age $(\mathrm{y} / \mathrm{o})$ & $58.1 \pm 0.8$ & DPP-4 inhibitors & $164(73.5 \%)$ \\
$\begin{array}{l}\text { Body } \\
\text { weight }(\mathrm{kg})\end{array}$ & $77.9 \pm 1.5$ & Metformin & $126(56.5 \%)$ \\
BMI $\left(\mathrm{kg} / \mathrm{m}^{2}\right)$ & $28.4 \pm 0.5$ & Sulfonylurea & $16(7.2 \%)$ \\
HbA1c $(\%)$ & $7.89 \pm 0.08$ & GLP-1 agonists & $10(4.5 \%)$ \\
LDL-cholesterol & $108.4 \pm 6.4$ & Insulin & $65(29.1 \%)$ \\
$(\mathrm{mg} / \mathrm{dL})$ & & & \\
& & Antihypertensives & \\
eGFR $(\mathrm{mL} / \mathrm{min} /$ & $N(\%)$ & RAS inhibitors & $114(51.1 \%)$ \\
$1.73 \mathrm{~m})^{2}$ & & & \\
$\geq 90$ & $44(19.7 \%)$ & ARB & $69(30.9 \%)$ \\
$90>\geq 60$ & $120(53.8 \%)$ & ACE inhibitors & $45(20.2 \%)$ \\
$60>\geq 45$ & $38(17.0 \%)$ & Ca channel blockers & $87(39.0 \%)$ \\
$45>\geq 30$ & $13(5.8 \%)$ & $\beta$ blockers & $45(20.2 \%)$ \\
$30>$ & $8(3.6 \%)$ & Diuretics & $22(9.9 \%)$ \\
& & Loop & $18(8.1 \%)$ \\
& & Thiazides & $4(1.8 \%)$ \\
\hline
\end{tabular}

inhibitors. Thirty-nine percent of the subjects received CCBs, of which amlodipine was used in more than half of the patients (amlodipine; $22.0 \%$, nifedipine CR; $6.7 \%$, cilnidipine; $4.5 \%$, azelnidipine; $3.6 \%$, benidipine; $2.2 \%$ ). $\beta$ blockers were given to $20.2 \%$ of the subjects at the beginning of the study.

Administration of SGLT2 inhibitors reduced HbA1c $(-0.53 \%, p<0.001)$, BW $(-2.6 \mathrm{~kg}, p<0.001)$ and systolic $\mathrm{BP}(-2.5 \mathrm{mmHg}, p<0.05)$ at 3 months, and these changes were sustained throughout the study period (Table 2). Serum $\mathrm{Mg}$ was elevated following treatment with SGLT2 inhibitors $(p<0.01)$.

SGLT2 inhibitors elicited a decrease in eGFR at 3 months (from $73.2 \pm 1.6$ to $69.5 \pm 1.6 \mathrm{~mL} / \mathrm{min} / 1.73 \mathrm{~m}^{2}$ $p<0.005$ ), and this effect was sustained until the end of the study (Table 2). The rate of the changes in eGFR from 3 to 12 months was $1.1 \pm 1.1 \mathrm{~mL} / \mathrm{min} / 1.73 \mathrm{~m}^{2} /$ year. The urinary albumin-to-creatinine ratio was nearly unchanged throughout the study period.

\section{Effects of various parameters on initial eGFR dips}

To elucidate what factors had a significant impact on the initial eGFR reduction induced by SGLT2 inhibitors, the correlations between the early decrease in eGFR ( 0 to 3 months) and various biochemical and anthropometric parameters were assessed with the use of stepwise multiple regression analysis. Thus, male sex, baseline eGFR and metformin use constituted significant determinants of the early changes in eGFR (male: $\beta=-8.541, p<0.005$; baseline eGFR: $\beta=0.189, p<0.005$; metformin use: $\beta=$ $6.406, p<0.05)$. CCB use was marginally associated with the initial dip in eGFR $(\beta=-5.780, p=0.07)$. In contrast, age or changes in systolic BP, BW and HbA1c were not correlated with early decreases in eGFR.

\section{Effects of antidiabetic drugs on SGLT2 inhibitor- induced changes in eGFR}

Background use of metformin significantly prevented the SGLT2 inhibitor-induced decrease in eGFR (Fig. 1a, e). Thus, under no metformin treatment at baseline, SGLT2 inhibitors reduced eGFR by $5.9 \pm 1.3 \mathrm{~mL} / \mathrm{min} / 1.73 \mathrm{~m}^{2}$ at 3 months (i.e., from $67.9 \pm 2.9$ to $61.8 \pm 2.5 \mathrm{~mL} / \mathrm{min} / 1.73$ $\left.\mathrm{m}^{2}, p<0.005\right)$. In metformin users, however, SGLT2 inhibitor-induced changes in eGFR were blunted $(77.9 \pm 1.7$ to $75.7 \pm 1.8 \mathrm{~mL} / \mathrm{min} / 1.73 \mathrm{~m}^{2}, p<0.05$ ), corresponding to approximately one-third of the response observed in patients who were not using metformin $(p=0.023)$. To eliminate the possibility that baseline renal function might affect eGFR responses to SGLT2 inhibitors, the effect of SGLT2 inhibitors on eGFR was assessed in patients with baseline eGFR $\geq 45 \mathrm{~mL} / \mathrm{min} / 1.73 \mathrm{~m}^{2}$; the baseline eGFR 
Table 2 Temporal changes in blood and kidney parameters
Fig. 1 Effects of antidiabetic drugs on SGLT2 inhibitorinduced changes in eGFR. SGLT2i; SGLT2 inhibitors, DPP4i; DPP4 inhibitors, SU; sulfonylurea, Met; metformin. $* p<0.05, * * p<0.01, * * * p<$ 0.005 vs. 0 month

\begin{tabular}{lllll}
\hline$N=223$ & Baseline & 3 months & 6 months & 12 months \\
\hline Body weight $(\mathrm{kg})$ & $77.9 \pm 1.5$ & $75.3 \pm 1.5^{* * *}$ & $75.7 \pm 1.6^{* * *}$ & $76.3 \pm 1.5^{*}$ \\
BMI $\left(\mathrm{kg} / \mathrm{m}^{2}\right)$ & $28.4 \pm 0.5$ & $27.6 \pm 0.5^{*}$ & $27.7 \pm 0.5^{*}$ & $27.8 \pm 0.4^{*}$ \\
Systolic BP $(\mathrm{mmHg})$ & $138.2 \pm 1.2$ & $135.7 \pm 1.4^{*}$ & $134.8 \pm 1.3^{*}$ & $133.3 \pm 1.4^{* *}$ \\
Diastolic BP $(\mathrm{mmHg})$ & $78.9 \pm 1.0$ & $77.4 \pm 1.1$ & $76.8 \pm 1.0$ & $76.6 \pm 1.0$ \\
HbA1c $(\%)$ & $7.89 \pm 0.08$ & $7.36 \pm 0.06^{* * *}$ & $7.36 \pm 0.06^{* * *}$ & $7.32 \pm 0.07^{* * *}$ \\
LDL-cholesterol $(\mathrm{mg} / \mathrm{dL})$ & $108.4 \pm 6.4$ & $99.9 \pm 2.0$ & $100.1 \pm 2.1$ & $99.8 \pm 2.1$ \\
Serum Mg $(\mathrm{mg} / \mathrm{dL})$ & $1.93 \pm 0.02$ & $2.07 \pm 0.02^{* *}$ & $2.05 \pm 0.02^{* *}$ & $2.01 \pm 0.02^{* *}$ \\
eGFR $\left(\mathrm{mL} / \mathrm{min} / 1.73 \mathrm{~m}^{2}\right)$ & $73.2 \pm 1.6$ & $69.5 \pm 1.6^{* * *}$ & $69.7 \pm 1.6^{* * *}$ & $70.2 \pm 1.7^{*}$ \\
UACR $(\mathrm{mg} / \mathrm{g})$ & $67.6(14.6-411.7)$ & $69.8(16.0-281.0)$ & $53.8(13.8-301.5)^{*}$ & $60.4(13.6-276.2)$ \\
\hline UACR, $\mathrm{urine}$ & & &
\end{tabular}

UACR, urine albumin/creatinine ratio

$* p<0.05, * * p<0.01, * * * p<0.005$ vs. baseline
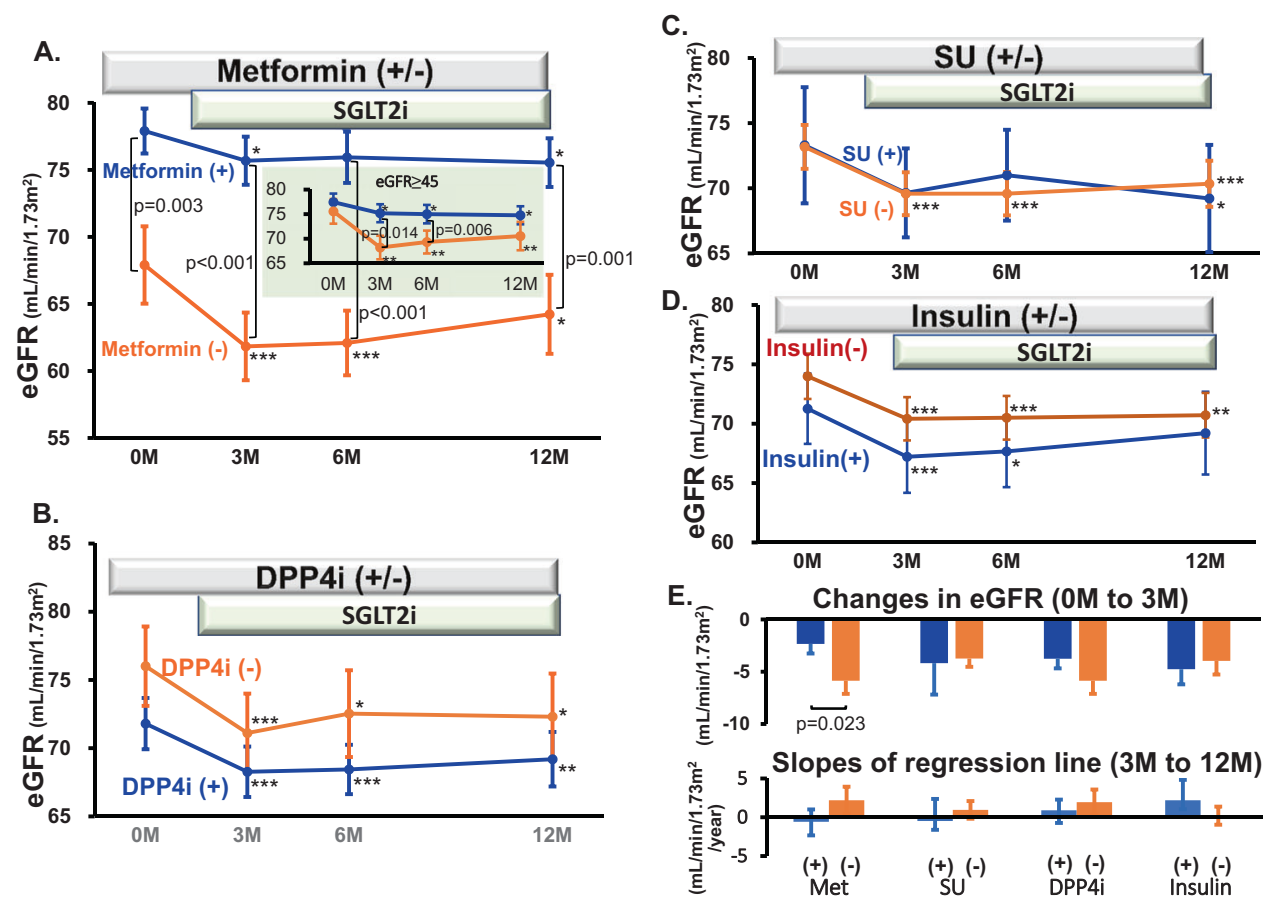

did not differ between metformin users and nonusers, and the blunted responses to SGLT2 inhibitors were again seen under metformin treatment. Changes in HbA1c, systolic BP or BW did not differ between these groups.

Other background medications, including DPP4i, sulfonylureas and insulin, had no effects on the changes in eGFR induced by SGLT2 inhibitors (Fig. 1b-e). None of the antidiabetic drugs used for background medications affected the long-term (3 to 12 months) changes in eGFR (Fig. 1e).

\section{Effects of antihypertensive drugs on SGLT2 inhibitor-induced changes in eGFR}

\section{RAS inhibitors}

Among 223 subjects, 114 patients had been given RAS inhibitors as background medications when SGLT2 inhibitors were started (Table 1). Baseline eGFR did not differ between the RAS inhibitor-treated and untreated groups (Fig. 2a). In subjects without RAS inhibitor treatment, the administration of SGLT2 inhibitors caused an initial decrease in eGFR from $74.6 \pm 2.3$ to $71.8 \pm 2.4 \mathrm{~mL} /$ $\mathrm{min} / 1.73 \mathrm{~m}^{2}$ at 3 months $(p<0.05)$, and the responses were sustained until the last visit. Under background treatment with RAS inhibitors, SGLT2 inhibitors also reduced eGFR, manifesting an initial decrease from $71.9 \pm 2.2$ to $67.4 \pm 2.0$ $\mathrm{mL} / \mathrm{min} / 1.73 \mathrm{~m}^{2}$ at 3 months $(p<0.005)$, which was marginally greater than that in patients not using RAS inhibitors ( $p=0.059$, Fig. $2 \mathrm{~d}$ ). When the effects of ACEis and ARBs on the SGLT2 inhibitor-induced changes in eGFR were compared, no difference was noted between these two subgroups, although the effect of ACEis tended to be greater than that of ARBs or non-RAS inhibitors at 3 months (Fig. 3a). 
Fig. 2 Effects of antihypertensive drugs on SGLT2 inhibitor-induced changes in eGFR. SGLT2i; SGLT2 inhibitors, RASi; reninangiotensin system inhibitors, $\mathrm{CCB} ; \mathrm{Ca}^{2+}$ channel blockers. $* p<0.05, * * p<0.01, * * * p<$ 0.005 vs. 0 month
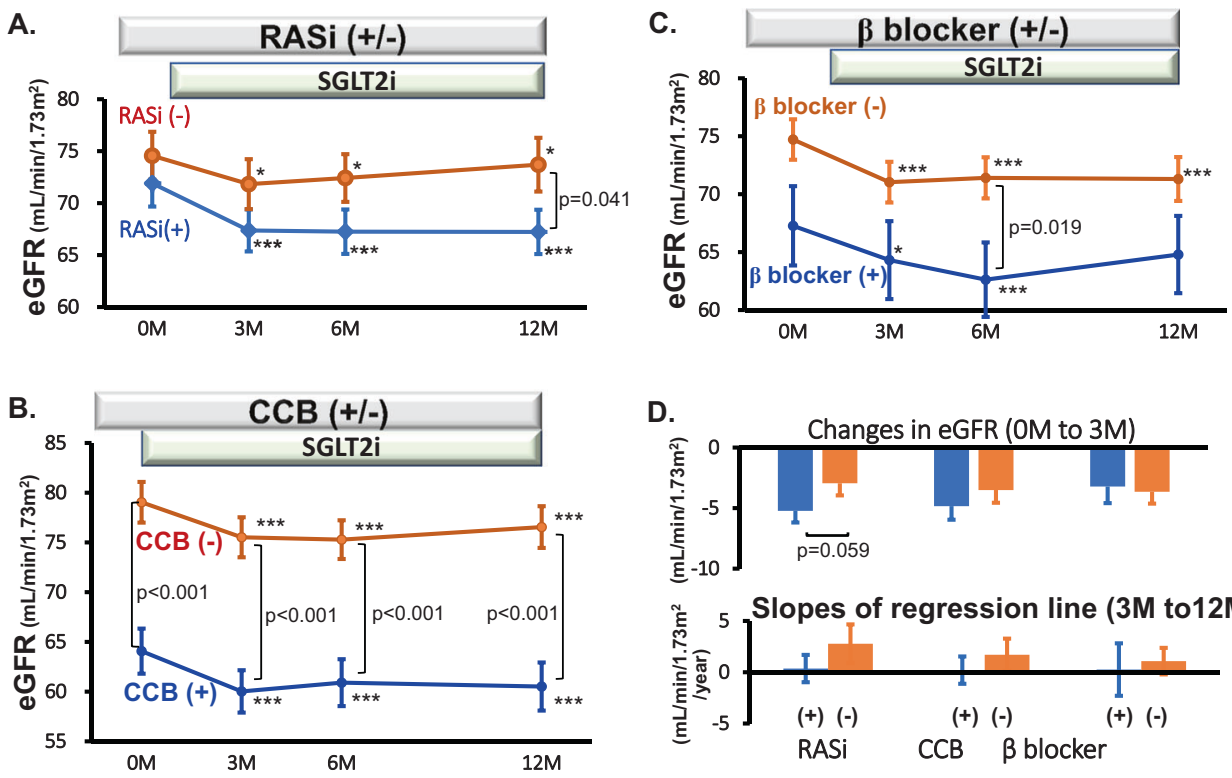

D.

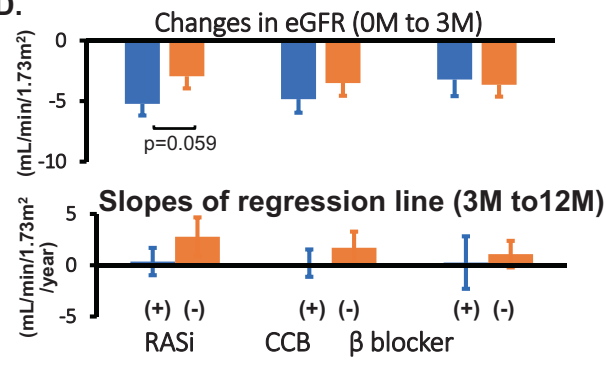

Fig. 3 Effects of ACE inhibitors/ ARB and Ca channel subtype blockers on SGLT2 inhibitorinduced changes in eGFR. ACEi; ACE inhibitors, ARB; angiotensin receptor blockers, $\mathrm{CCB} ; \mathrm{Ca}^{2+}$ channel blockers. Non-RASi; subjects not using ACEi or ARB. Non-CCB: subjects not using $\mathrm{Ca}^{2+}$ channel blockers. ${ }^{*} p<0.05$, $* * p<0.01$, $* * * p<0.005$ vs. 0 month

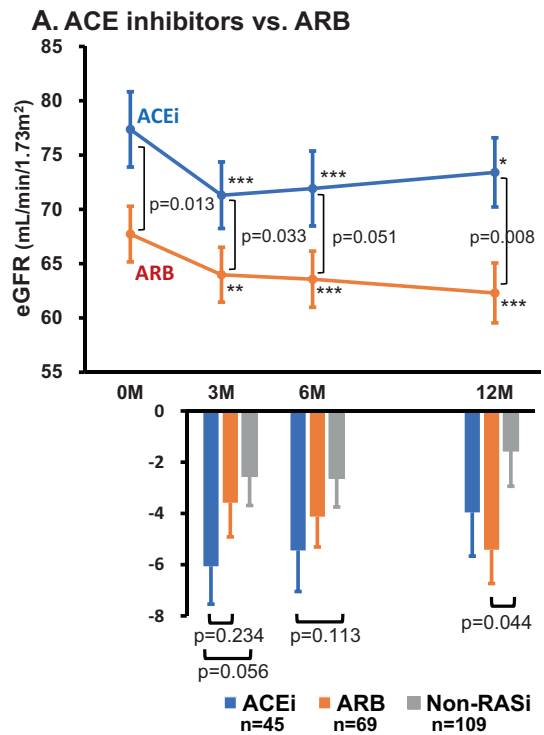

B. L- vs. N- vs. T-type CCB

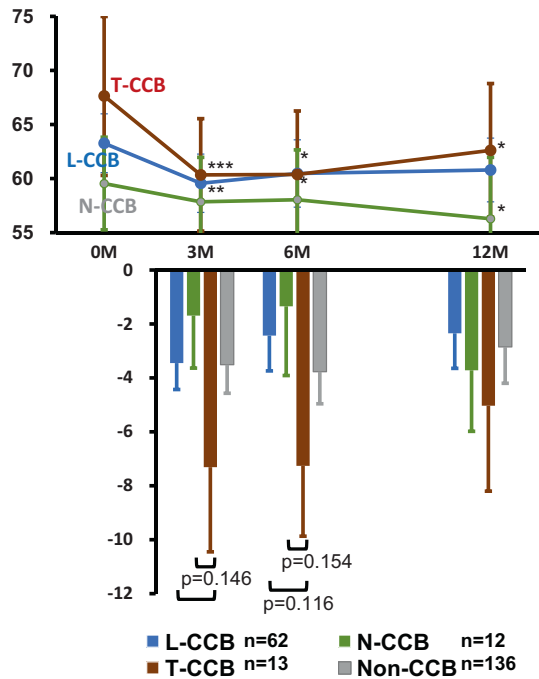

\section{CCBs}

Baseline eGFR was significantly lower in patients treated with CCBs than in those not given CCBs $(p<0.001$, Fig. 2b). In patients using CCBs as background therapy at baseline, SGLT2 inhibitors substantially reduced eGFR from $64.1 \pm 2.3$ to $60.0 \pm 2.1 \mathrm{~mL} / \mathrm{min} / 1.73 \mathrm{~m}^{2}$ at 3 months $(p<0.005)$. The SGLT2 inhibitor-induced changes in eGFR did not differ between the CCB-treated and untreated groups (Fig. 2d). Furthermore, to eliminate the confounding effect of different baseline eGFRs on the subsequent responses to SGLT2 inhibitors, the subjects were categorized into the groups with the following baseline eGFRs: $\geq 90,<90$ and $\geq 60,<60$ and $\geq 45,<45$. Thus, no difference in the baseline eGFR or the response to SGLT2 inhibitors was seen between the CCB-treated and untreated groups for any of the eGFR categories (Supplementary Fig.).

CCBs are subclassified as L-, T- and N-type CCBs [11]. When the background effects of CCB subtypes on SGLT2 inhibitor-induced changes in eGFR were evaluated, the reduction in eGFR tended to be more pronounced under treatment with T-type CCBs than under L- or N-type CCB treatment (Fig. 3b). 
Fig. 4 Interaction between SGLT2 inhibitors and antidiabetic/antihypertensive drugs on eGFR after 3 months of SGLT2 inhibitor therapy. SGLT2i; SGLT2 inhibitors, RASi; renin-angiotensin system inhibitors, $\mathrm{CCB} ; \mathrm{Ca}^{2+}$ channel blockers, DPP4i; DPP4 inhibitors

\section{A. Metformin vs. RAS inhibitors}

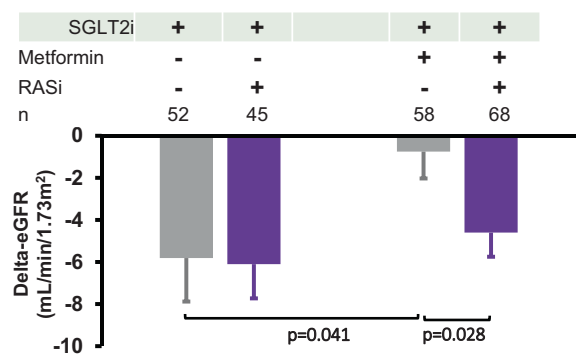

C. Metformin vs. insulin

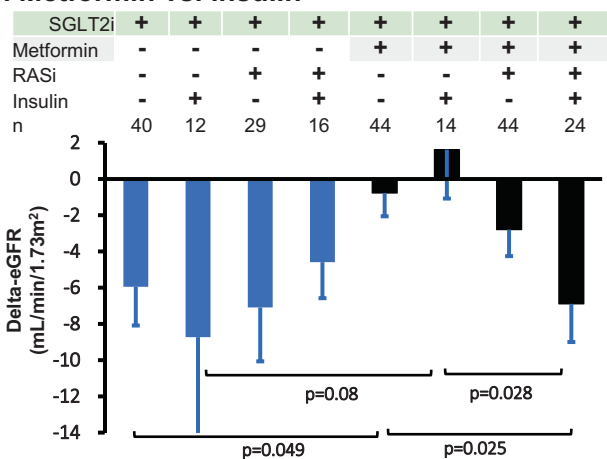

B. Metformin vs. antihypertensive drugs

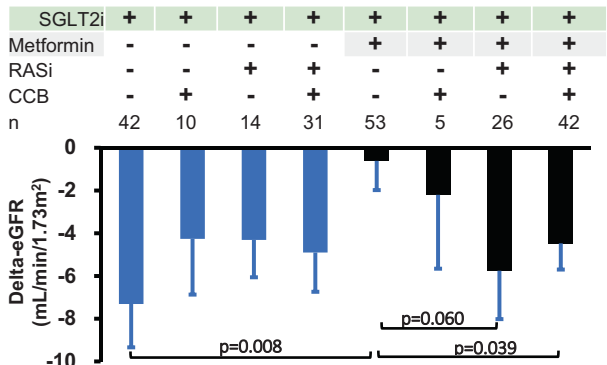

D. Metformin vs. DPP4 inhibitors
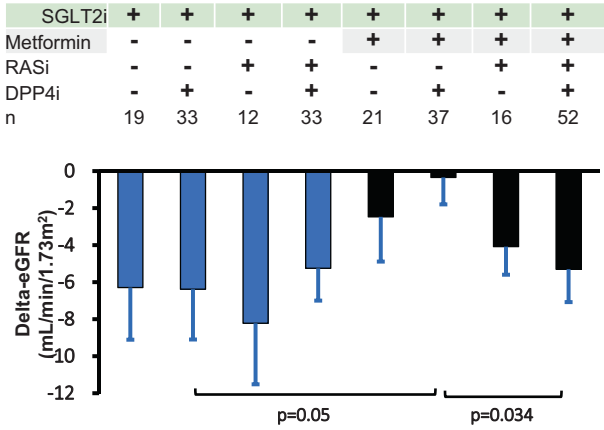

\section{$\beta$-blockers}

In patients who had been treated with $\beta$ blockers at baseline, the administration of SGLT2 inhibitors reduced the eGFR from $67.3 \pm 3.4$ to $64.3 \pm 3.4 \mathrm{~mL} / \mathrm{min} / 1.73 \mathrm{~m}^{2}$ at 3 months $(p<0.05$, Fig. 2c). The changes in eGFR by SGLT2 inhibitors did not differ from those in patients not using $\beta$ blockers $\left(-3.22 \pm 1.37\right.$ vs. $-4.17 \pm 0.86 \mathrm{~mL} / \mathrm{min} / 1.73 \mathrm{~m}^{2}$, $p>0.5$, Fig. 2d).

None of the background antihypertensive medications, including RAS inhibitors, CCBs and $\beta$ blockers, modified the long-term ( 3 to 12 months) effects of SGLT2 inhibitors on eGFR (Fig. 2d).

\section{Interactions among antidiabetic and antihypertensive drugs}

The interactive effects of antidiabetic and antihypertensive drugs on the changes in eGFR were assessed after 3 months of SGLT2 inhibitor treatment. In patients not taking metformin as a background medication, SGLT2 inhibitors decreased eGFR, which did not depend on concomitant use of RAS inhibitors (Fig. 4a). In contrast, under background treatment with metformin, the eGFR response to SGLT2 inhibitors was abolished in patients not using RAS inhibitors (from $79.9 \pm 2.3$ to $79.3 \pm 2.7 \mathrm{~mL} / \mathrm{min} / 1.73 \mathrm{~m}^{2}, n=58$, $p>0.5)$ but was preserved when RAS inhibitors had been given along with metformin for baseline therapy (from 75.9 \pm 2.4 to $72.4 \pm 2.4 \mathrm{~mL} / \mathrm{min} / 1.73 \mathrm{~m}^{2}, n=68, p<0.01$ ).

We further evaluated the effects of metformin, RAS inhibitors and CCBs on the eGFR responses to SGLT2 inhibitors (Fig. 4b). In patients not taking metformin, RAS inhibitors or CCBs as background medication at baseline, SGLT2 inhibitors caused a decrease of $7.3 \pm 2.0 \mathrm{~mL} / \mathrm{min} /$ $1.73 \mathrm{~m}^{2}$ in eGFR (i.e., from $71.7 \pm 4.8$ to $63.9 \pm 4.4 \mathrm{~mL} /$ $\left.\min / 1.73 \mathrm{~m}^{2}, n=42, p<0.001\right)$. Likewise, the initial eGFR dip seen in patients using CCBs, RAS inhibitors or both but not metformin at baseline did not differ from that with SGLT2 inhibitors alone. In striking contrast, under background treatment with metformin but not CCBs or RAS inhibitors, SGLT2 inhibitors failed to reduce eGFR (from $81.0 \pm 2.3$ to $80.6 \pm 2.8 \mathrm{~mL} / \mathrm{min} / 1.73 \mathrm{~m}^{2}, n=53, p>0.5$ ). Treatment with CCBs, along with metformin, did not cause significant improvement in the SGLT2 inhibitor-induced initial eGFR dip, but RAS inhibitors marginally recovered this response $(p=0.06)$. The combination of all three medications at baseline significantly restored the SGLT2 inhibitor-induced response of eGFR.

The interaction between SGLT2 inhibitors and other antidiabetic drugs (i.e., metformin, insulin and DPP4 inhibitors) was assessed after 3 months of SGLT2 inhibitor treatment. Again, among these antidiabetic drugs as background medications, only metformin, but not insulin or DPP4 inhibitors, affected the SGLT2 inhibitor-induced decrease in eGFR, which was restored by RAS inhibitors (Fig. 4c, d).

\section{Discussion}

Abundant evidence has accrued indicating that SGLT2 inhibitors not only ameliorate glycemic control but also 
offer beneficial action on several vital organs, including the heart, liver and kidneys. Recent randomized trials, such as EMPA-REG OUTCOME [3], the CANVAS Program [4], DECLARE-TIMI58 [5] and CREDENCE [6], suggest that early reductions in eGFR following treatment with SGLT2 inhibitors are possibly mediated by enhanced TGF and are supposedly associated with improved renal outcomes in type 2 DM patients. In these trials, however, multiple antidiabetic and antihypertensive drugs were used as background medications, which could modify the renal hemodynamic action of SGLT2 inhibitors and might influence long-term renal outcomes. For example, in the EMPA-REG OUTCOME, CANVAS and CREDENCE trials, 80 to $100 \%$ of the subjects had received RAS inhibitors prior to the administration of SGLT2 inhibitors [3-6]. Likewise, metformin was used in 50 to $80 \%$ of the patients in combination with SGLT2 inhibitors. It appears relevant therefore to clarify the role of concomitant use of antidiabetic and antihypertensive drugs in the SGLT2 inhibitorinduced renal hemodynamic effects in patients with type 2 DM and hypertension.

The present study shows that SGLT2 inhibitors not only ameliorate glycemic control but also alter renal hemodynamics (Table 2). Furthermore, SGLT2 inhibitors reduce systemic BP and BW and increase serum $\mathrm{Mg}$, which is consistent with the results demonstrated previously [3$6,12]$. In our current study, the patients received several classes of antidiabetic drugs, including DPP4 inhibitors (73.5\%), metformin (56.5\%), and insulin (29.1\%), as background medications at baseline. We therefore evaluated the impact of these antidiabetic drugs on the eGFR responses to SGLT2 inhibitors. Thus, DPP4 inhibitors, sulfonylureas or insulin as background medication had no effect on the SGLT2 inhibitor-induced changes in eGFR (Fig. 1). In contrast, metformin markedly suppressed the renal hemodynamic response to SGLT2 inhibitors. Furthermore, to eliminate the possible confounding effects of RAS inhibitors and CCBs, the SGLT2 inhibitor-induced changes in eGFR were evaluated under the condition where no RAS inhibitor or CCB was given; metformin almost totally abolished the SGLT2 inhibitor-induced changes in eGFR $\left(-0.61 \pm 1.37 \mathrm{~mL} / \mathrm{min} / 1.73 \mathrm{~m}^{2}\right.$, Fig. 4b). Several studies showed that the administration of metformin elevated eGFR [13, 14]. Furthermore, Rosenstock et al. [14] demonstrated that the SGLT2 inhibitor-induced reduction in eGFR was attenuated by the simultaneous administration of metformin, which agreed with our results and hence suggested the vasodilator property of metformin. In this regard, it is recognized that metformin stimulates AMP-activated protein kinase (AMPK) [15], which would facilitate nitric oxide production $[16,17]$. The background treatment with metformin may therefore blunt the action of SGLT2 inhibitor-induced TGF via nitric oxide [18] and could lead to the diminished response of eGFR to SGLT2 inhibitors. Of note, van Bommel et al. [19] recently demonstrated that the dapagliflozin-induced activation of TGF and the decrease in eGFR are attributed to efferent arteriolar vasodilation rather than afferent arteriolar constriction in metformin-treated patients with type 2 DM. Thus, the efferent arteriolar dilation induced by SGLT2 inhibitors could be undermined under the condition where this arteriole is already dilated by metformin.

Although it remains fully undetermined to what extent the renal hemodynamic action of SGLT2 inhibitors contributes to improved renal outcomes in type 2 DM [20], metformin interference with the eGFR response to SGLT2 inhibitors might affect the progressive nature of DM nephropathy. Metformin per se is reported to cause beneficial [21] or detrimental effects on renal function in patients with type 2 DM $[13,22]$. It has been demonstrated, however, that in the EMPA-REG OUTCOME trial, empagliflozin reduces the risks of progressive nephropathy but induces a diminished reduction in this risk for patients using metformin compared with those not using metformin $(32 \%$ vs. $53 \%$ risk reduction, interaction $P=0.01$ ) [8]. Similar observations have been reported concerning the cardioprotective effects of empagliflozin (29\% vs. 54\% risk reduction of cardiovascular death, interaction $P=0.07$ ) [8] and canagliflozin ( $12 \%$ vs. $36 \%$ risk reduction of cardiovascular death or hospitalization, interaction $P=0.03$ ) [9]. In this regard, Packer $[23,24]$ suggested that the beneficial effects of SGLT2 inhibitors that depend on AMPK are attenuated in the setting where AMPK has already been activated by background treatment with metformin. This interactive effect is noteworthy because combination therapy with metformin and SGLT2 inhibitors is commonly provided to a large number of patients with type 2 DM [3, 4, 6]. Nevertheless, even under background therapy with metformin, SGLT2 inhibitors should still offer substantial renal and cardiovascular benefits. Furthermore, therapeutic strategies for treating patients with DM and hypertension actually include RAS inhibitors [25, 26], which could restore the metformin-induced changes in renal hemodynamic action (Fig. 4).

RAS inhibitors are widely used in patients with DM and hypertension and are regarded as a first-line antihypertensive drug for DM [25, 26]. This class of agents exerts favorable action on renal outcomes, which is mediated at least in part by the amelioration of glomerular hypertension ascribed to efferent arteriolar dilation [27]. Similarly, SGLT2 inhibitors have emerged as a promising antidiabetic drug that confers additional benefits to alleviate progressive kidney disease, whose renal action is purportedly attributed in part to afferent arteriolar vasoconstriction due to enhanced TGF [28-30]. Intriguingly, van Bommel et al. [19] recently showed efferent arteriolar vasodilation 
by dapagliflozin as a crucial mechanism for reduced glomerular pressure in metformin-treated patients with type 2 DM; two-thirds of the patients had been given RAS inhibitors, and dapagliflozin caused decreases in both renal vascular resistance and GFR. It is anticipated therefore that the concomitant use of SGLT2 inhibitors and RAS inhibitors could act in concert to modulate the efferent arteriolar tone, resulting in the improvement in glomerular hypertension. In this regard, the post hoc analysis of the EMPAREG OUTCOME by Mayer et al. [31] showed that in patients taking RAS inhibitors as a background medication at baseline, empagliflozin elicited a greater initial eGFR change than in those not taking RAS inhibitors. The present study also shows that background treatment with RAS inhibitors marginally enhances the SGLT2 inhibitor-induced decrease in eGFR (Fig. 2). Furthermore, under background treatment with metformin, the SGLT2 inhibitor-induced decrease in eGFR was abolished, but this response was efficiently preserved when RAS inhibitors had been given along with metformin for baseline therapy (Fig. 4a). Of note, it is well known that adenosine constitutes a crucial mediator of TGF, eliciting not only preglomerular vasoconstriction but also postglomerular vasodilation [32-34], especially in the presence of RAS inhibitors [32]. Collectively, these observations suggest an intimate interplay between SGLT2 inhibitors and RAS inhibitors acting on glomerular microvessels, whereby the renal hemodynamic effect of SGLT2 inhibitors is augmented.

Of interest, the present study shows that ACEis tend to augment the SGLT2 inhibitor-induced decrease in eGFR to a greater extent than ARBs (Fig. 3). It is well known that ACEi stimulates bradykinin activity [35], and we previously demonstrated that an ACEi caused increased vasodilator action on efferent arterioles than an ARB in dog kidneys, the mechanism of which involved enhanced intrarenal bradykinin activity [36]. Nevertheless, the long-term effects of these RAS inhibitors on SGLT2 inhibitor-induced changes in renal function need to be established.

CCB is a potent antihypertensive agent and is accepted as an add-on therapy to RAS inhibitors in hypertensive patients with DM. In the EMPA-REG OUTCOME trial, $27.8 \%$ to $45.8 \%$ of the patients received CCBs [3]. Because CCBs potently cause afferent arteriolar vasodilation [11,37], CCBs could counter the afferent arteriolar constriction induced by SGLT2 inhibitor-induced augmentation of TGF. The post hoc analysis for the EMPA-REG OUTCOME, however, shows that CCBs do not modify the empagliflozin-induced decrease in eGFR [31]. Similarly, our present study demonstrates that treatment with CCBs has no inhibitory effect on the SGLT2 inhibitor-induced decrease in eGFR, irrespective of background medication with RAS inhibitors or metformin (Figs. 2 and 4). These observations are somewhat surprising because CCBs should render afferent arterioles refractory to the constrictor action of TGF. Of note, TGF has been shown to affect not only afferent but also efferent arteriolar tone in experimental animals, whose effects are mediated by intrarenal adenosine generation and the subsequent stimulation of adenosine $\mathrm{A} 1$ and $\mathrm{A} 2$ receptors [32-34]. Adenosine constricts afferent arterioles through adenosine A1 receptor-mediated stimulation of phospholipase C/inositol trisphosphate (IP3) production [38]. To the extent that IP3 stimulates Ca release from the sarcoplasmic reticulum and opening of $\mathrm{Ca}^{2+}$-activated chloride channels, leading to depolarization and $\mathrm{Ca}$ influx through voltagedependent $\mathrm{Ca}$ channels [38-40], CCBs should block this vasoconstrictor mechanism. In contrast, efferent arterioles are devoid of L-type voltage-dependent $\mathrm{Ca}$ channels, a target for CCBs [11], but manifest vasodilation by adenosine through adenosine A2 receptors [32-34]. Because SGLT2 inhibitors increase urinary adenosine excretion in patients with type 2 [19] as well as type 1 DM [30, 41], adenosine could be responsible for SGLT2 inhibitor-induced efferent arteriolar vasodilation. Indeed, a recent study clearly shows that the SGLT2 inhibitor-induced decrease in eGFR is caused by postglomerular vasodilation rather than preglomerular vasoconstriction in metformin-treated type $2 \mathrm{DM}$ patients [19]. It is judiciously anticipated therefore that SGLT2 inhibitors have the ability to decrease eGFR under the influence of CCBs.

Several subclasses of CCBs have been documented depending on the activity of different $\mathrm{Ca}$ channel subtypes. Nifedipine and amlodipine preferentially block L-type $\mathrm{Ca}$ channels and inhibit renal preglomerular (e.g., afferent) arteriolar tone [11]. Furthermore, efonidipine, azelnidipine and benidipine affect both preglomerular and postglomerular (i.e., efferent) arteriolar tone through blockade of L-type and T-type Ca channels [11, 42-44]. Finally, cilnidipine inhibits $\mathrm{N}$-type $\mathrm{Ca}$ channels in the nerve terminals innervating afferent and efferent arterioles and hence dilates both microvessels $[11,44]$. In the present study, we found a modest tendency of T-type CCBs to enhance the SGLT2i-induced reduction in eGFR (Fig. 3). Because of the paucity of patients treated with T-type and N-type CCBs, however, the interaction between $\mathrm{CCB}$ subtypes and SGLT2 inhibitors needs to be more thoroughly elucidated.

The present study also evaluated the influence of $\beta$ blockers on eGFR responses to SGLT2 inhibitors. The results show that $\beta$ blockers have no effect on the SGLT2 inhibitor-induced decrease in eGFR (Fig. 2). Furthermore, this effect was again blunted by background therapy with metformin (from $74.6 \pm 3.9$ to $72.7 \pm 3.7 \mathrm{~mL} / \mathrm{min} / 1.73 \mathrm{~m}^{2}$, $n=22, p=0.375$ ) versus the absence of metformin (from $60.6 \pm 5.2$ to $56.3 \pm 5.1 \mathrm{~mL} / \mathrm{min} / 1.73 \mathrm{~m}^{2}, n=23, p=$ 0.013). Other parameters, including $\mathrm{HbAlc}$ and $\mathrm{BP}$, exhibited similar changes in response to SGLT2 inhibitors in the $\beta$ blocker-treated and untreated groups. 
Finally, the present study attempted to evaluate the effect of SGLT2 inhibitors on albuminuria and its association with changes in eGFR. Because of the nature of this study protocol (e.g., retrospective study), numerous missing data on albuminuria preclude its precise evaluation. Furthermore, since SGLT2 inhibitors upregulate sirtuin-1 (SIRT1) [45] and then mitigate glomerular injury through nonhemodynamic mechanisms, including the suppression of claudin-1 [46] and restored function of autophagy [47], hemodynamic parameters (i.e., eGFR and glomerular hypertension) may not necessarily parallel the renal pathophysiological process in DM nephropathy. Additionally, although the slopes of long-term (3 to 12 months) changes in eGFR do not differ between users and nonusers of specific background drugs, the relatively short observation period of this study (i.e., 12 months) abates the accuracy in assessing the temporal changes in renal function. More extensive studies would clarify the effect of SGLT2 inhibitors on albuminuria and long-term renal outcomes and could more precisely unveil the interaction of SGLT2 inhibitors with other antidiabetic and antihypertensive drugs.

In conclusion, the present study shows that background treatment with antidiabetic and antihypertensive drugs modifies the renal hemodynamic effects of SGLT2 inhibitors in patients with type $2 \mathrm{DM}$. Whereas the concomitant use of metformin and SGLT2 inhibitors confers greater benefits on glycemic control, there is a possibility that preceding treatment with metformin may diminish the SGLT2 inhibitor-induced decrease in eGFR. Further addition of RAS inhibitors to background medications, by contrast, restores this response. Finally, CCBs, though potent afferent arteriolar vasodilators, have no impact on the SGLT2 inhibitor-induced change in eGFR. Whether the renal hemodynamic effects of these background medications are linked to long-term renal outcomes requires further investigation.

\section{Compliance with ethical standards}

Conflict of interest The authors declare that they have no conflict of interest.

Publisher's note Springer Nature remains neutral with regard to jurisdictional claims in published maps and institutional affiliations.

Open Access This article is licensed under a Creative Commons Attribution 4.0 International License, which permits use, sharing, adaptation, distribution and reproduction in any medium or format, as long as you give appropriate credit to the original author(s) and the source, provide a link to the Creative Commons license, and indicate if changes were made. The images or other third party material in this article are included in the article's Creative Commons license, unless indicated otherwise in a credit line to the material. If material is not included in the article's Creative Commons license and your intended use is not permitted by statutory regulation or exceeds the permitted use, you will need to obtain permission directly from the copyright holder. To view a copy of this license, visit http://creativecommons. org/licenses/by/4.0/.

\section{References}

1. Prischl FC, Wanner C. Renal outcomes of antidiabetic treatment options for type 2 diabetes-a proposed MARE definition. Kidney Int Rep. 2018;3:1030-8.

2. Groop PH, Cooper ME, Perkovic V, Emser A, Woerle HJ, von Eynatten M. Linagliptin lowers albuminuria on top of recommended standard treatment in patients with type 2 diabetes and renal dysfunction. Diabetes Care. 2013;36:3460-8.

3. Wanner C, Inzucchi SE, Lachin JM, Fitchett D, von Eynatten M, Mattheus M, et al. Empagliflozin and progression of kidney disease in type 2 diabetes. N. Engl J Med. 2016;375:323-34.

4. Perkovic V, de Zeeuw D, Mahaffey KW, Fulcher G, Erondu N, Shaw W, et al. Canagliflozin and renal outcomes in type 2 diabetes: results from the CANVAS Program randomised clinical trials. Lancet Diabetes Endocrinol. 2018;6:691-704.

5. Fioretto P, Stefansson BV, Johansson E, Cain VA, Sjostrom CD. Dapagliflozin reduces albuminuria over 2 years in patients with type 2 diabetes and renal impairment. Diabetologia. 2016;59: 2036-9.

6. Perkovic V, Jardine MJ, Neal B, Bompoint S, Heerspink HJL, Charytan DM, et al. for the CREDENCE Trial Investigators. Canagliflozin and renal outcomes in type 2 diabetes and nephropathy. N. Engl J Med. 2019;380:2295-306.

7. Cherney DZI, Zinman B, Elnzucchi SE, Koitka-Weber A, Mattheus M, von Eynatten M, et al. Effects of empagliflozin on the urinary albumin-to-creatinine ratio in patients with type 2 diabetes and established cardiovascular disease: an exploratory analysis from the EMPA-REG OUTCOME randomised, placebocontrolled trial. Lancet Diabetes Endocrinol 2017;5:610-21.

8. Inzucchi SE, Fitchett D, Jurisic-Erzen D, Woo V, Hantel S, Janista $\mathrm{C}$, et al. on behalf of the EMPA-REG OUTCOME Investigators. Are the cardiovascular and kidney benefits of empagliflozin influenced by baseline glucose-lowering therapy? Diabetes Obes Metab. 2020;22:631-9.

9. Radholm K, Figtree G, Perkovic V, Solomon SD, Mahaffey KW, de Zeeuw D, et al. Canagliflozin and heart failure in type 2 diabetes mellitus. Circulation. 2018;138:458-68.

10. Matsuo S, Imai E, Horio M, Yasuda Y, Tomita K, Nitta K, et al. Revised equations for estimated GFR from serum creatinine in Japan. Am J Kidney Dis. 2009;53:982-92.

11. Hayashi K, Wakino S, Sugano N, Ozawa Y, Homma K, Saruta T. $\mathrm{Ca}^{2+}$ channel subtypes and pharmacology in the kidney. Circ Res. 2007;100:342-53.

12. Tang H, Zhang X, Zhang J, Li Y, Del Gobbo LC, Zhai S, et al. Elevated serum magnesium associated with SGLT2 inhibitor use in type 2 diabetes patients: a meta-analysis of randomised controlled trials. Diabetologia. 2016;59:2546-51.

13. Lachin JM, Viberti G, Zinman B, Haffner SM, Aftring RP, Paul $\mathrm{G}$, et al. Renal function in type 2 diabetes with rosiglitazone, metformin, and glyburide monotherapy. Clin J Am Soc Nephrol. 2011;6:1032-40.

14. Rosenstock J, Chuck L, Gonzalez-Ortiz M, Merton K, Craig J, Capuano G, et al. Initial combination therapy with canagliflozin plus metformin versus each component as monotherapy for drugnaïve type 2 diabetes. Diabetes Care. 2016;39:353-62.

15. Ren H, Shao Y, Wu C, Ma X, Lv C, Wang Q. Metformin alleviates oxidative stress and enhances autophagy in diabetic kidney disease via AMPK/SIRT-1-FoxO1 pathway. Mol Cell Endocrinol. 2020;500:110628. 
16. Cho KJ, Casteel DE, Prakash P, Tan L, van der Hoeven D, Salim AA. et al. AMPK and endothelial nitric oxide synthase signaling regulates K-Ras plasma membrane interactions via cyclic GMPdependent protein kinase 2. Mol Cell Biol. 2016;36:3086-99.

17. Sambe T, Mason RP, Dawoud H, Bhatt DL, Malinski T. Metformin treatment decreases nitroxidative stress, restores nitric oxide bioavailability and endothelial function beyond glucose control. Biomed Pharmacother. 2018;98:149-56.

18. Carlstrom M, Wilcox CS, Arendshorst WJ. Renal autoregulation in health and disease. Physiol Rev. 2015;95:405-511.

19. van Bommel EJM, Muskiet MHA, van Baar MJB, Tonneijck L, Smits MM, Emanuel AL, et al. The renal hemodynamic effects of the SGLT2 inhibitor dapagliflozin are caused by post-glomerular vasodilatation rather than pre-glomerular vasoconstriction in metformin-treated patients with type 2 diabetes in the randomized, double-blind RED trial. Kidney Int. 2020;97:202-12. https://doi. org/10.1016/j.kint.2019.09.013

20. Kohagura K, Yamasaki H, Takano H, Ohya Y, Seino Y. Luseogloflozin, a sodium-glucose cotransporter2 inhibitor, preserves renal function irrespective of acute changes in the estimated glomerular filtration rate in Japanese patients with type 2 diabetes. Hypertens Res. 2020;43:876-83.

21. Hippislay-Cox J, Coupland C. Diabetes treatment and risk of amputation, blindness, severe kidney failure, hyperglycemia, and hypoglycemia: open cohort study in primary care. BMJ. 2016; 352:i1450.

22. Hsu WH, Hsiao PJ, Lin PC, Chen SC, Lee MY, Shin SJ. Effect of metformin on kidney function in patients with type 2 diabetes mellitus and moderate chronic kidney disease. Oncotarget. 2018;9:5416-23.

23. Packer M. Does metformin interfere with the cardiovascular benefits of SGLT2 inhibitors? Questions about its role as the cornerstone of diabetes treatment. Am J Med 2020. S0002-9343 (20)30112-1. https://doi.org/10.1016/j.amjmed.2020.01.016

24. Packer M. Role of impaired nutrient and oxygen deprivation signaling and deficient autophagic flux in diabetic CKD development: implications for understanding the effects of sodium-glucose cotransporter 2-inhibitors. J Am Soc Nephrol. 2020;31:907-19.

25. Cosentino F, Grant PJ, Aboyans V, Bailey CJ, Ceriello A, Delgado V, et al. ESC Scientific Document Group. 2019 ESC guidelines on diabetes, pre-diabetes, and cardiovascular diseases developed in collaboration with the EASD: The Task Force for diabetes, pre-diabetes, and cardiovascular diseases of the European Society of Cardiology (ESC) and the European Association for the Study of Diabetes (EASD). Eur Heart J. 2020;41:255-323.

26. Umemura S, Arima H, Arima S, Asayama K, Dohi Y, Hirooka Y, et al. Japanese society of hypertension committee for guidelines for the management of hypertension. The Japanese society of hypertension guidelines for the management of hypertension (JSH 2019). Hypertens Res. 2019;49:1235-481.

27. Brenner BM, Hostetter TH, Olson JL, Rennke HG, Venkatachalam MA. The role of glomerular hyperfiltration in the initiation and progression of diabetic nephropathy. Acta Endocrinol Suppl (Copenh). 1981;242:7-10.

28. Kidokoro K, Cherney DZI, Bozovic A, Nagasu H, Satoh M, Kanda E. et al. Evaluation of glomerular hemodynamic function by empagliflozin in diabetic mice using in vivo imaging. Circulation. 2019;140:303-15.

29. Heerspink HJL, Perkins BA, Fitchett DH, Husain M, Cherney DZI. Sodium glucose cotransporter 2 inhibitors in the treatment of diabetes mellitus. Circulation. 2016;134:752-72.
30. Cherney DZ, Perkins BA, Soleymanlou N, Maione M, Lai V, Lee A, et al. Renal hemodynamic effect of sodium-glucose cotransporter 2 inhibitor in patients with type 1 diabetes mellitus. Circulation. 2014;129:587-97.

31. Mayer G, Wanner C, Weir MR, Inzucchi SE, Koitka-Weber A, Hantel S, et al. Analysis from the EMPA-REG OUTCOME trial indicates empagliflozin may assist in preventing the progression of chronic kidney disease in patients with type 2 diabetes irrespective of medications that alter intrarenal hemodynamics. Kidney Int. 2019;96:489-504.

32. Vallon V, Muhlbauer B, Osswald H. Adenosine and kidney function. Physiol Rev. 2006;86:901-40.

33. Ren Y, Garvin JL, Liu R, Carretero OA. Possible mechanism of efferent arteriole (Ef-Art) tubuloglomerular feedback. Kidney Int 2007;71:861-6.

34. Al-Mashhadi RH, Skott O, Vanhoutte PM, Hansen PB. Activation of A2 adenosine receptors dilates cortical efferent arterioles in mouse. Kidney Int 2009;75:793-9.

35. Chen K, Zimmerman BG. Comparison of renal hemodynamic effect of ramipril to captopril: possible role of kinins. J Pharm Exp Ther. 1994;270:491-7.

36. Matsuda H, Hayashi K, Arakawa K, Naito M, Kubota E, Honda $\mathrm{M}$, et al. Zonal heterogeneity in action of angiotensin-converting enzyme inhibitor on renal microcirculation: role of intrarenal bradykinin. J Am Soc Nephrol. 1999;10:2272-82.

37. Loutzenhiser R, Epstein M, Hayashi K. Renal hemodynamic effects of calcium antagonists. Am J Cardiol. 1989;64:41F-45F.

38. Hansen PB, Friis UG, Uhrenholt TR, Briggs J, Schnermann J. Intracellular signalling pathways in the vasoconstrictor response of mouse afferent arterioles to adenosine. Acta Physiol (Oxf) 2007;191:89-97.

39. Takenaka T, Suzuki H, Kanno Y, Fujiwara K, Hayashi K, Nagahama $\mathrm{T}$, et al. Cellular mechanisms mediating rat renal microvascular constriction by angiotensin II. J Clin Invest. 1997;100:2107-14.

40. Nagahama T, Hayashi K, Ozawa Y, Takenaka T, Saruta T. Role of protein kinase $\mathrm{C}$ in angiotensin II-induced constriction of renal microvessels. Kidney Int. 2000;57:215-23.

41. Rajasekeran H, Lytvyn Y, Bozovic A, Lovshin JA, Diamandis E, Cattran D, et al. Urinary adenosine excretion in type 1 diabetes. Am J Physiol 2017;313:F184-91.

42. Hayashi K, Nagahama T, Oka K, Epstein M, Saruta T. Disparate effects of calcium antagonists on renal microcirculation. Hypertens Res. 1996;19:31-6.

43. Hayashi K, Ozawa Y, Fujiwara K, Wakino S, Kumagai H, Saruta T. Role of actions of calcium antagonists on efferent arterioles -with special references to glomerular hypertension -. Am J Nephrol. 2003;23:229-44.

44. Hayashi K, Ozawa Y, Saruta T, Epstein M: Renal hemodynamic effects of calcium antagonists. In Calcium Antagonists in Clinical Medicine ( $3^{r d} e d$.). eds. by Epstein M, Hanley \& Belfus, pp. 559-578, 2002.

45. Umino H, Hasegawa K, Minakuchi H, Muraoka H, Kawaguchi T, Kanda T. et al. High basolateral glucose increases sodium-glucose cotransporter 2 and reduces sirtuin-1 in renal tubules through glucose transporter-2 detection. Sci Rep. 2018;8:6791

46. Hasegawa K, Wakino S, Simic P, Sakamaki Y, Minakuchi H, Fujimura K, et al. Renal tubular Sirt1 attenuates diabetic albuminuria by epigenetically suppressing Claudin-1 overexpression in podocytes. Nat Med. 2013;19:1496-504.

47. Kume $S$, Thomas MC, Koya D. Nutrient sensing, autophagy, and diabetic nephropathy. Diabetes. 2012;61:23-9. 